\title{
AVALIAÇÃO IN VITRO DE FITOTERÁPICOS NO CONTROLE DE RHIPICEPHALUS BOOPHILUS MICROPLUS
}

\author{
NICOLINO, Carlos Augusto ${ }^{1}$ \\ PARANHOS, Rafael Mendonça ${ }^{2}$ \\ MACIEL, Willian ${ }^{1}$
}

\begin{abstract}
RESUMO: O uso de fitoterápicos é considerado uma alternativa importante no controle de parasitas, e podem reduzir impactos econômicos e ambientais ao uso de pesticidas sintéticos. O presente estudo teve por objetivo avaliar comparativamente a ação in vitro de óleos essenciais contra fêmeas ingurgitadas de Rhipicephalus (Boophilus) microplus. Foi utilizado óleo essencial de Azadiracta indica (Neem), Cymbopogom nardus L. Rendle (Citronela) e Cymbopogon citratus (Capim-cidreira) nas seguintes concentrações: 12,5\%, 6\%, 3\% e 1\%. Eficácia máxima (100\%) foi observada por todas as plantas nas concentrações de $12,5 \%$. Na concentração de $6 \%$ somente os óleos essenciais de neem e citronela apresentaram eficácia de $100 \%$. A citronela ainda apresentou eficácia máxima na concentração de $3 \%$ e ainda obteve $95,30 \%$ na menor concentração (1\%). Conclui-se que a utilização de óleos essenciais pode ser uma alternativa importante no controle de Rhipicephalus (Boophilus) microplus.
\end{abstract}

Palavras-chave: Fitoterápico. Carrapato. Rhipicephalus Boophilus microplus.

SUMMARY: The use of herbal medicines is considered an important alternative to control pests, and can reduce economic and environmental impacts the use of synthetic pesticides. The present study aimed at comparing the in vitro action of essential oils against engorged females of Rhipicephalus (Boophilus) microplus. Essential oil was used Azadiracta indica (Neem), Cymbopogom nardus L. Rendle (Citronella) and Cymbopogon citratus (lemon-grass) in the following concentrations: $12.5 \%, 6 \%, 3 \%$ and $1 \%$. Maximum efficiency $(100 \%)$ was observed for all plants at concentrations of $12.5 \%$. At the concentration of $6 \%$ only the essential oils of neem and citronella apresntaram $100 \%$ efficiency. Citronella still showed maximum efficiency at a concentration of 3\% and still got $95.30 \%$ in the lowest concentration (1\%). It is concluded that the use of essential oils can be an important alternative in the control of Rhipicephalus (Boophilus) microplus.

Keywords: Herbal medicine. Tick. Rhipicephalus Boophilus microplus.

\section{INTRODUÇÃO}

A infestação por carrapato Rhipicephalus (Boophilus) microplus é uma das doenças parasitárias de maior impacto econômico nos rebanhos de bovinos leiteiros. É encontrado em áreas tropicais e subtropicais entre os paralelos $32^{\circ}$ norte e $32^{\circ}$ sul com alguns focos nos paralelos $35^{\circ}$ norte e sul. Atualmente é encontrado na Ásia, Austrália, México, América Central, América do Sul e África (KESSLER ; SCHENK, 1998).

Estratégias de controle desses parasitos, normalmente, enfocam a utilização de produtos carrapaticidas sintéticos, rotação de pastagens e uso de tratamentos em períodos estratégicos. Porém o rápido aparecimento de carrapatos resistentes aos produtos convencionais (FURLONG et al., 2004), especialmente em rebanhos de bovinos leiteiros (OLIVEIRA ; AZEVEDO, 2002), associado ao alto custo do tratamento, além de resíduos em alimentos e à poluição ambiental causada por sua utilização, incentivam a pesquisa de alternativas, como os fitoterápicos.

\footnotetext{
${ }^{1}$ Médico Veterinário/Pós-graduando Faculdade de Ciências Agrárias e Veterinárias FCAV/UNESP Campus de Jaboticabal

${ }^{2}$ Médico Veterinário/Professor Dr. Universidade de Franca / Faculdade Dr. Francisco Maeda.
} 
Diante do exposto, o aproveitamento de fitoterápicos tem sido uma alternativa importante no controle de ácaros, podendo diminuir os impactos econômicos, ambientais e consequentemente o uso de pesticidas sintéticos. Embora ainda pouco pesquisado, as soluções feitas com extratos de plantas tendem apresentar menor toxicidade aos mamíferos, rápida degradação e lerdo desenvolvimento de resistência.

No entanto, interesse pelo estudo das plantas medicinais vem crescendo nos últimos anos, principalmente, no que se refere à sua atividade farmacêutica e composição química. Tais fatores incentivaram a busca por outras formas de controle, mais incisivamente a partir da década de 90 . Dentre essas, o uso de fitoterápicos destaca-se devido à grande volubilidade de espécies existentes, baixo custo, fácil disponibilidade na propriedade e, principalmente, pela baixa ou ausência de contaminação do ambiente e, em consequência, dos animais e do homem.

O Brasil possui 55.000 espécies de plantas aproximadamente, é considerado o País com o maior número de espécies no mundo, estudos sobre possíveis efeitos terapêuticos dessas plantas são muito reduzidos (DI STASI, 1996). O mesmo autor relata que poucas informações são conhecidas sobre a composição química de $99,6 \%$ das plantas da flora Nacional.

Para Araújo Filho (2000), a fitoterapia e a homeopatia são bases para o controle de doenças na produção animal ecológica, trazendo a vantagem do melhor retorno econômico pelo menor desembolso com a compra de produtos químicos industrializados, não deixando resíduos tóxicos contaminantes, além de terem demonstrado resultados na prevenção e na cura dessas doenças.

No início de janeiro de 2009, o Ministério da Saúde divulgou uma lista com 71 plantas que podem ser utilizadas como medicamento fitoterápico, assim em conformidade com as plantas contidas a lista, utilizando-se do Neem (Azadiracta indica A. Juss), do capim-cidreira (Cymbopogon citratus) e da citronela (Cymbopogon nardus L. Rendle), parte a pesquisa para comprovar a eficácia. Dentre as plantas indicadas para o controle de insetos destacam-se Azadiracta indica A. Juss, o capim-cidreira (Cymbopogon citratus) e a citronela (Cymbopogon nardus L. Rendle).

Diante disso o presente trabalho estudou-se a eficácia in vitro de óleos essenciais de diferentes plantas no controle do carrapato bovino Riphicephalus (B.) microplus.

\section{MATERIAL E MÉTODOS}

O experimento ocorreu no laboratório da FAFRAM - Faculdade Doutor Francisco Maeda, localizada no município de Ituverava - SP. Foram utilizados exemplares de uma mesma estirpe de Rhipicephalus (Boophilus) microplus provenientes de colônia isolada e mantida no CPPAR/FCAVJ/UNESP Campus de Jaboticabal/SP. Para a realização dos testes foram utilizados os seguintes óleos essenciais extraídos por meio de arraste de vapor: Azadiracta indica (Neem), Cymbopogon citratus (Capim-cidreira) e Cymbopogon nardus L. Rendle (Citronela).

As fêmeas de Rhipicephalus (B.) microplus foram submetidas às concentrações de 12,5\%,6\%, 3\% e $1 \%$ de óleo essencial das plantas supracitadas, diluídas em água destilada. O grupo controle foi banhado com água destilada, conforme sumarizado no quadro a seguir: 


\begin{tabular}{|c|c|c|}
\hline Planta medicinal & Concentrações & $\mathbf{N}^{\mathbf{0}}$ de Teleógenas \\
\hline $\begin{array}{c}\text { Azadiracta indica } \text { (Neem) } \\
\text { Cymbopogon citratus } \\
\text { (Capim-cidreira) }\end{array}$ & $12,5 \%, 6 \%, 3 \%, 1 \%$ & 10 \\
\hline $\begin{array}{c}\text { Cymbopogon nardus L. Rendle } \\
\text { (Citronela) }\end{array}$ & $12,5 \%, 6 \%, 3 \%, 1 \%$ & 10 \\
\hline Placebo Controle & $12,5 \%, 6 \%, 3 \%, 1 \%$ & 10 \\
\hline
\end{tabular}

Foi utilizada a metodologia preconizada por DRUMMOND et al. (1971). Fêmeas ingurgitadas, sem qualquer anomalia ou mutilação visível, foram colhidas após desprenderem-se de bovinos, posteriormente foram lavadas em água destilada. Para cada um dos quatro grupos experimentais foram selecionadas 10 fêmeas, agrupadas pelo peso, sendo em seguida imersas em $100 \mathrm{~mL}$ de cada solução, durante cinco minutos, utilizando-se copos de plástico descartáveis. Decorrido este tempo, os grupos de fêmeas ingurgitadas foram recolhidos e o excesso de solução retirado com papel toalha. Posteriormente, as fêmeas foram fixadas com fita adesiva dupla face, em placa de Petri devidamente identificadas e mantidas em estufa B.O.D.

Após 15 dias, a massa de ovos presente em cada placa de Petri foi pesada e transferida para seringas plásticas adaptadas, vedadas com algodão, e mantidas em estufa B.O.D., nas mesmas condições previamente citadas. Ao final da eclosão das larvas, foi avaliado o percentual de eclodibilidade.

Para o cálculo da Eficiência Reprodutiva foi aplicado a seguinte fórmula (DRUMMOND et al., 1973):

Eficiência Reprodutiva $(E R)=\ldots$ Peso dos ovos $(\mathrm{g}) \quad$ x $\%$ Eclosão x $27000^{1}$

Peso das Teleóginas (g)

A eficácia do produto de cada um dos fármacos empregados foi calculada por meio da seguinte fórmula (DRUMMOND et al., 1973):

Eficácia do Produto $=\underline{E R}($ grupo controle $)-E R($ grupo tratado $) \times 100$

ER (grupo controle)

\section{RESULTADOS}

Considerando que a eficácia de um produto carrapaticida deve mostrar uma efetividade de acima de 95\%, os resultados da avaliação com óleo essencial de capim-cidreira (Tabela 1) atingiram eficácia superior a 95\% somente na concentração de $12,5 \%$, por outro lado à utilização desta concentração poderia tornar o custo do produto inviável, além disso os carrapaticidas devem ser altamente efetivos contra todos os estágios evolutivos dos parasitos, serem inócuos para o animal e o homem, não devem contaminar o ambiente e não devem ter efeito cancerígeno nem mutagênico. (CORDOVÉS, 1997). 
Tabela 1.Performace reprodutiva de fêmeas de Rhipicephalus Boophilus microplus (Cepa campo CPPAR), submetidas a ação de óleos essenciais (Cidreira) fitoterápicos. Ituverava, SP, Brasil.

\begin{tabular}{|c|c|c|c|c|c|c|c|}
\hline Princípo ativo & Grupo & & Teleógina & Peso da Postura (G) & | Percentual de Eclodibilidade & Eficiência Reprodutiva & Eficácia Produto \\
\hline & & $\mathrm{N}$ & Peso (g) & & & & \\
\hline Controle (água destilada) & GIA & 10 & 2,58 & 0,73 & 96,3 & 543460,47 & .. \\
\hline Óleo essencial de cidreira 12,5\% & GIIA & 10 & 1,52 & 0 & 0 & 0 & 100 \\
\hline Óleo essencial de cidreira $6 \%$ & GIIIA & 10 & 2,15 & 0,1 & 84 & 78139,53 & 85,62 \\
\hline Óleo essencial de cidreira $3 \%$ & GIVA & 10 & 2,00 & 0,38 & 66,6 & 251082,01 & 53,8 \\
\hline óleo essencial de cidreira 1\% & GVA & 10 & 2,13 & 0,39 & 91,3 & 332623,47 & 38,8 \\
\hline
\end{tabular}

Com relação ao uso do neem no controle de carrapatos, verifica-se pela Tabela 2 que a ação do óleo essencial contra fêmeas ingurgitadas de Rhipicephalus (Boophilus) microplus obteve eficácia de $100 \%$ em duas diluições: $12,5 \%$ e $6 \%$. Nas concentrações de $3 \%$ e $1 \%$ o óleo desta planta apresentou eficácia moderada, $88,36 \%$ e $70,94 \%$ respectivamente.

Tabela 2.Performace reprodutiva de fêmeas de Rhipicephalus Boophilus microplus (Cepa campo CPPAR), submetidas a ação de óleos essenciais (Neem) fitoterápicos. Ituverava, SP, Brasil.

\begin{tabular}{|c|c|c|c|c|c|c|c|}
\hline Principo ativo & Grupo & & Teleógina & Peso da Postura (G) & Percentual de Eclodibilidade & Eficiência Reprodutiva & Eficácia Produto \\
\hline & & N & Peso (g) & & & & \\
\hline Controle (água destilada) & GIA & 10 & 2,58 & 0,73 & 96,3 & 543460,47 & \\
\hline Óleo essencial de cidreira 12,5\% & GIIA & 10 & 1,92 & 0,05 & 0 & 0 & 100 \\
\hline Óleo essencial de cidreira $6 \%$ & GIIIA & 10 & 1,80 & 0,042 & 0 & 0 & 100 \\
\hline óleo essencial de cidreira 3\% & GIVA & 10 & 1,79 & 0,123 & 48,6 & 63257,14 & 88,36 \\
\hline óleo essencial de cidreira $1 \%$ & GVA & 10 & 1,82 & 0,237 & 57,3 & 157908,14 & 70,94 \\
\hline
\end{tabular}

O óleo extraído das sementes de Neem teve sua eficácia demonstrada in vitro contra carrapatos da família Ixodidae (Ndumu et al., 1999). Embora utilizando extrato alcoólico Borges et al. (2003), também observaram efeitos similares do neem contra fêmeas ingurgitadas de Rhipicephalus (Boophilus) microplus, encontrando índices de eficácia de até $100 \%$.

Analisando a Tabela 3, observa-se que o óleo essencial de citronela apresentou eficácia máxima (100\%) nas concentrações de 12,5\%, 6\% e 3\% e mesmo na concentração de $1 \%$ obteve alta eficácia, 95,30\% contra fêmeas ingurgitadas de Rhipicephalus (Boophilus) microplus. Estes resultados apresentam grande similaridade com os observado por Olivo al.; 2008, que encontraram 92,1\% de eficácia do o óleo de citronela sobre fêmeas ingurgitadas de Rhipicephalus (Boophilus) microplus, na concentração de $1 \%$. 
Tabela 3.Performace reprodutiva de fêmeas de Rhipicephalus Boophilus microplus (Cepa campo CPPAR), submetidas a ação de óleos essenciais (Citronela) fitoterápicos. Ituverava, SP, Brasil.

\begin{tabular}{|l|l|lr|c|c|c|c|c|}
\hline Principo ativo & Grupo & \multicolumn{3}{c}{ Teleógina Peso da Postura (G) Percentual de Eclodibilidade Eficiência Reprodutiva Eficácia Produto } \\
\hline Controle (água destilada) & GIA & 10 & 2,58 & 0,73 & 96,3 & 543460,47 & \\
\hline Óleo essencial de cidreira 12,5\% & GIIA & 10 & 2,65 & 0 & 0 & 0 & 100 \\
\hline Óleo essencial de cidreira 6\% & GIIIA & 10 & 2,38 & 0 & 0 & 0 & 100 \\
\hline Óleo essencial de cidreira 3\% & GIVA & 10 & 2,51 & 0 & 0 & 0 & 100 \\
\hline Óleo essencial de cidreira 1\% & GVA & 10 & 2,70 & 0,66 & 52,3 & 25568,89 & 95,3 \\
\hline
\end{tabular}

Considerando-se os resultados obtidos com as soluções feitas com extratos de plantas, que tendem a apresentar menor toxicidade aos mamíferos, rápida degradação e desenvolvimento lento de resistência (CHUNGSAMARNYART ; JIWAJINDA, 1992), estes resultados podem ser importantes como estratégia de controle do carrapato em diferentes sistemas de produção.

\section{CONCLUSÃO}

Por meio do delineamento experimental proposto, pode se extrair as seguintes inferências: As formulações utilizando óleos essenciais de fitoterápicos podem constituir importantes alternativas para uma diminuição das infestações de carrapatos. Constata-se, no entanto, a necessidade de se realizar novos estudos para otimizar a utilização de óleos essenciais, especialmente na avaliação com emulsionantes, na forma e no período de utilização do produto constituído.

\section{REFERÂNCIAS}

ARAÚJO FILHO, R. Introdução à pecuária ecológica: a arte de criar animais sem drogas ou venenos. Porto Alegre: São José, 2000. 136p.

CHUNGSAMARNYART, N.; JIWAJINDA, S. Acaricidal activity of volatile oil from lemon and citronella grasses on tropical cattle ticks. Kasetsart Journal, v.26, p.46-51, 1992.

CORDOVÉS, C. O. Carrapato: controle ou erradicação. Guaíba: Agropecuária, 1997.

DI STASI, L. C. Plantas medicinais: arte e ciência. Um guia de estudo interdisciplinar. São Paulo: Ed. da UNESP, 1996. 230p.

DRUMMOND, R. O. et al. Boophilus annulatus and B. microplus: laboratory tests of insecticides.

Journal of Economic Entomology, v. 66, n. 1, p. 130-133, 1971.

FURLONG, J. et al., Controle estratégico do carrapato dos bovinos. A Hora Veterinária, RS, v.23, n.137, p. 53-56, 2004.

KESSLER, R.H., SCHENCK, M. A. M. Carrapato, tristeza parasitária e tripanossomose dos bovinos. Campo Grande: EMBRAPA-CNPGC, 1998. 157p.

NDUMU, PA.; GEORGE, J.B.D.; CHOUDHURY, M.K. Toxicity of Neem Seed Oil (Azadirachta indica) against the larvae of Amblyimma variegatu a three-host tick in cattle. Phytot. Res., v.13, p.532-534, 1999. 
OLIVEIRA, A. A., AZEVEDO, H. C. Resistência do carrapato Boophilus microplus a carrapaticidas em bovinos de leite na região dos tabuleiros costeiros de Sergipe. Revista Científica Rural, v.7, n.2, p.64-71, 2002.

OLIVO, C.J.et al. [Alcoholic extract of lemongrass (Cymbopogon citratus) on the control of

Boophilusmicroplus in cattle]. Revista Brasileira de Parasitologia Veterinária, v.15, n.1. p.37-39, 2006. 